\title{
PALABRAS DE PATRICE VERMIEREN EN REPRESENTACIÓN DEL DIRECTOR-GENERAL DE LA UNESCO, FEDERICO MAYOR (AYUNTAMIENTO DE BARCELONA, 31 DE MARZO DE 1996)
}

Si je prends la parole ici et maintenant, en tant que représentant de Monsieur Federico Mayor, Directeur général de l'UNESCO et comme philosophe membre de la Division de la Philosophie de cette Organisation, c'est d'abord parce qu'à l'occasion du cinquantième anniversaire de l'Organisation, son Directeur général a voulu montrer l'extrême importance qu'il accordait à la philosophie et à son enseignement dans le monde actuel. L'UNESCO a toujours entretenu avec la philosophie un rapport de proximité. Les idéaux régulateurs qui commandent son Acte constitutif, en 19.45, puisent aux sources des traditions philosophiques. Et dès sa fondation, l'Organisation fait appel a la philosophie pour les mettre en oeuvre. On peut en effet lire dans son Préambule: "Les guerres prenant naissance dans l'esprit des hommes, c'est dans l'esprit des hommes que doivent être élevées les dếfenses de la paix». C'est dire qu'il faut faire confiance à la force des idées. au pouvoir de l'esprit humain, lorsqu'il est instruit et éclairé, pour maintenir la paix et la sécurité et assurer le respect universel de la justice, de la loi, des droits de l'homme et des libertés fondamentales pour tous. Dès sa fondation, l'UNESCO se propose en conséquence deux buts en matière de philosophie: 1) mettre des instruments internationaux adéquats au service de l'avancement des études philosophiques; 2) mettre la plilosophie au service de l'éducation intemationale des peuples en lui faisant jouer un röle fondamental dans la formation de l'esprit public.

C'est à cette source que puise Federico Mayor lorsqu'il écrit en 1995 dans la préface du livre de Roger-Pol Droit qui publie les résultats de l'enquête internationale que l'UNESCO vient de consacrer à la philosophie et à la démocratie dans le monde: «La contribution des philosophes est essentielle, à mes yeux, pour comprendre notre présent et pour construire les sociétés de demain. C'est pourquoi j'ai décidé de veiller personnellement au développement des activités de l'UNESCO dans le domaine philosophiquen. Et il décrit les deux raisons principales qui expliquent cette conviction: 1) la philosophie est une école de liberte: elle incite la pensée à s'éveiller toujours, contre la routine et par la création de concepts, et il faut qu'elle se confronte aux grands problèmes qui se posent aujourd'hui à l'humanité. 2) la philosophie, apprenant à juger par soi-mème, à disputer des argumentations diverses, à respecter la parole des autres, à se soumettre seulement à l'autorité de la raison, contribue de manière essentielle à la formation de citoy'ens libres

En ceci, si je puis me permettre, le Directeur général de l'UNESCO est très cartésien. Car Descartes s'est confrontc aux progrìs des sciences qui ont marqué son temps, singulièrement les mathématiques et l'optique; parce qu ${ }^{\mathrm{il}}$ a ouvert en métaphysique un nouveau régime de vérité, fondé sur la seule évidence de la Raison; en tant en fin que Descartes met en lumière la capacité égale en chaque ètre humain de faire usage de sa raison.

Votre Congrès présent, avec ses deux cents communications, a exploré systématiquement ces voies, et bien d'autres qui marquent l'actualité de Descartes. Il avait a priori toutes les raisons de recevoir le patronage de l'UNESCO. Le Directeur général avait inscrit la célébration du quatre centième anniversaire de Descartes au titre des commémorations auxquelles devait s'associer l'Organisation et il avait déjà prêté toute son attention au Premier Congrès international d`Ontologic organisé par le Professeur Victor Gomez-Pin à San Sebastian en avril 1993. au regard de la richesse et de la diversité de la réflexion philosophique au présent qui s'y śtait manifestće, en prenant comme prétexte Aristote. 
Mais a posteriori, votre Second Congrès mérite à un autre titre le patronage de l'UNESCO. Car il est sans doute un événement proprement philosophique. Qu'est-ce qu'un événement ? Un événement marque une rupture significative qui autorise la mise en opposition d'un avant et d'un après. Et pour mesurer l'importance d'un événement, il faut définir un critère d'ćvaluation qui permette de distinguer ce moment dans chaîne la temporelle des existants. Un événement n'est pas un accident; il a une cause dont on est susceptible de rendre raison. Si la Révolution française est un événement pour la philosophique de Kant, c'est parce qu'elle signe le moment de la réalisation de la liberté politique dans l'histoire du genre humain.

L'événement que constitue votre Congrès ne saurait certes être de même nature que la Révolution française pour Kant. Mais il a à bien des ćgards la signification historique d'une révolution dans les réunions de philosophes du temps. D'abord parce qu'il montre en acte la communauté philosophique espagnole toute entière rassemblée; dans l'expérience de sa pluralité, et dans l'accueil de philosophes venus du monde entier, ensuite par la capacité qui s'y est manifestée de conjoindre et de confronter les études les plus érudites et les plus rigoureuses de la philosophie de Descartes et de sa tradition avec les interrogations les plus contemporaines sur les savoirs scientifiques et techniques et des interrogations morales et politiques de notre temps, soit un Descartes revisité. Peut-être jamais mieux qu'ici n'a été rendu visible que Descartes n'appartient pas à la France, mais à la communauté universelle des esprits.

Pour avoir su dresser cette scène philosophique internationale inédite, Monsieur Victor Gomez-Pin doit être remercié. Avec ses collègues, ses amis, ses étudiants, avec Joan Rovira, Directeur du Département de Philosophie de l'Universitẻ Autonome de Barcelone, et Nicanor Ursua, Directeur du Département de Philosophie de l'Université du Pays Basque, il a permis que nous nous interrogions d'une manière décisive sur cette question radicale : comment permettre à la philosophie de jouer un rôle dans l'esprit humain ?

Cette question n'est pas seulement une interrogation théorique, elle a aussi des eflets pratiques. Qu'on me permette de faire ici allusion au combat que mène la communauté philosophique de ce pays pour défendre la place de sa discipline dans les programmes de l'enseignement secondaire et supérieur. Le monde moderne tend souvent à réduire, au nom d'impératifs économiques ou techniques à courte vue, l'enseignement de matières jugées inutiles. Il faut savoir qu'il existe une «Déclaration de Paris de la Philosophie», adoptée les 15 et 16 février 1995 à I'UNESCO, par tous les philosophes rassemblés aux Journées intemationales d'étude «Philosophie et démocratie dans le monden, et qui constitue pour la communauté philosophique internationale une charte à laquelle chacun peut se référer.

Votre Congrès a su, aux yeux de cette communauté philosophique internationale, témoigner du réveil du philosophique en Espagne. Il a montré aussi que Descartes n'appartient pas à une nation, à une culture, à un temps : il nous a prouvé qu'il était vivant.

Je voudrais enfin, à titre personnel, parce que je suis un spécialiste de la philosophie du xixè siècle, manifester aussi ma gratitude à Monsieur le Professeur René Thom, pour avoir montré l"intérêt contemporain de Charles Renouvier et de sa lecture du «cogito».

L'UNESCO vous remercie et croyez bien que sa Division de la Philosophie saura être au rendez-vous des prochains événements que le travail de réflexion de votre communauté plailosophique a d'ores et déjà annoncés dans le domaine de la pensée. 